hereditary angioneurotic edema. Acontribution to the early diagnosis of a disease of interdisciplinary significance. UltraschallMed. 1987;8:278-82.

4. Cicardi M, Bergamaschini L, Marasini B, Boccassini G, Tucci A, Agostoni A.Hereditary angioedema: an appraisal of 104 cases. Am J Med Sci. 1982;284:2-9. 5.Zingale LC, Zanichelli A, Deliliers DL, Rondonotti E, De Franchis R, Cicardi M.Successful resolution of bowel obstruction in a patient with hereditary angioedema. Eur JGastroenterol Hepatol. 2008;20:583-7.

\section{Inward migration of fully covered SEMS in walled off pancreatic necrosis}

Pancreatic fluid collections (PFCs) are an important local complication of acute pancreatitis that are associated with significant morbidity. ${ }^{1}$ Pseudocyst and walled off pancreatic necrosis (WOPN) are the delayed consequences of these collections. The pancreatic pseudocyst is defined as an encapsulated collection containing minimal solid material, whereas WOPN is an encapsulated collection of solid necrotic material with varying amount of liquid content. ${ }^{1}$ This distinction is important as patients with WOPN usually require more aggressive endoscopic drainage, in contrast to simple transmural drainage which may be adequate for treatment of pseudocysts..$^{2-5}$ The aggressive endoscopic drainage involves use of multiple plastic stents, fully covered self-expanding metallic stents (FC-SEMS) or direct endoscopic necrosectomy (DEN). The SEMS offer theoretical advantages, including a larger lumen diameter that allows easy DEN as well as need of fewer procedures. Stent migration is a well known complication of FC-SEMS and it is usually outward migration. In this case report, we report an inward migration of SEMS that was successfully retrieved endoscopically.

\section{Case Report}

A 34 year old male with a large $18 \mathrm{~cm}$ walled off pancreatic necrosis consequent to alcohol related acute necrotizing pancreatitis presented with abdominal pain and fever. He underwent endoscopic ultrasound (EUS) guided transmural drainage using a fully covered selfexpanding metallic stent (FC-SEMS) with wide flared ends (NAGI stent, Taewoong Medical Co., Ltd., Seoul, Korea). The WOPN decreased in size (Figure 1) but pain and fever persisted. Therefore, the patient was taken up for direct endoscopic necrosectomy. On endoscopy, the stent was not visualized (Figure 2) and fluoroscopy revealed migration of SEMS into WOPN cavity (Figure 3). The endoscope was pushed into the cavity over steel guide wire and stent could be seen inside the necrotic cavity (Figure 4). The retrieval string of the stent was identified and grasped with rat tooth forceps (Figure 5). The stent was gradually pulled back (Figure 6) and repositioned across the stomach (Figure 7) establishing transluminal drainage. Thereafter, endoscopic necrosectomy was done and patient had marked resolution of symptoms after 3 session of necrosectomy. The SEMS was removed 6 weeks later and replaced with two $10 \mathrm{Fr}$ double pigtail plastic stents. Thereafter, patient was discharged and is asymptomatic on follow up.

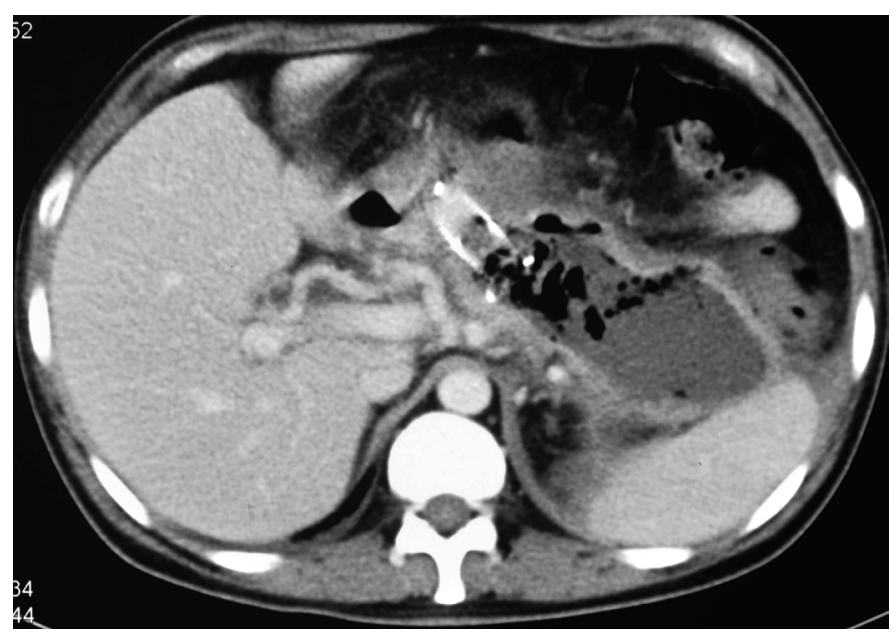

Figure 1: Computed tomography: Partially resolved WOPN with FC-SEMS in situ. 


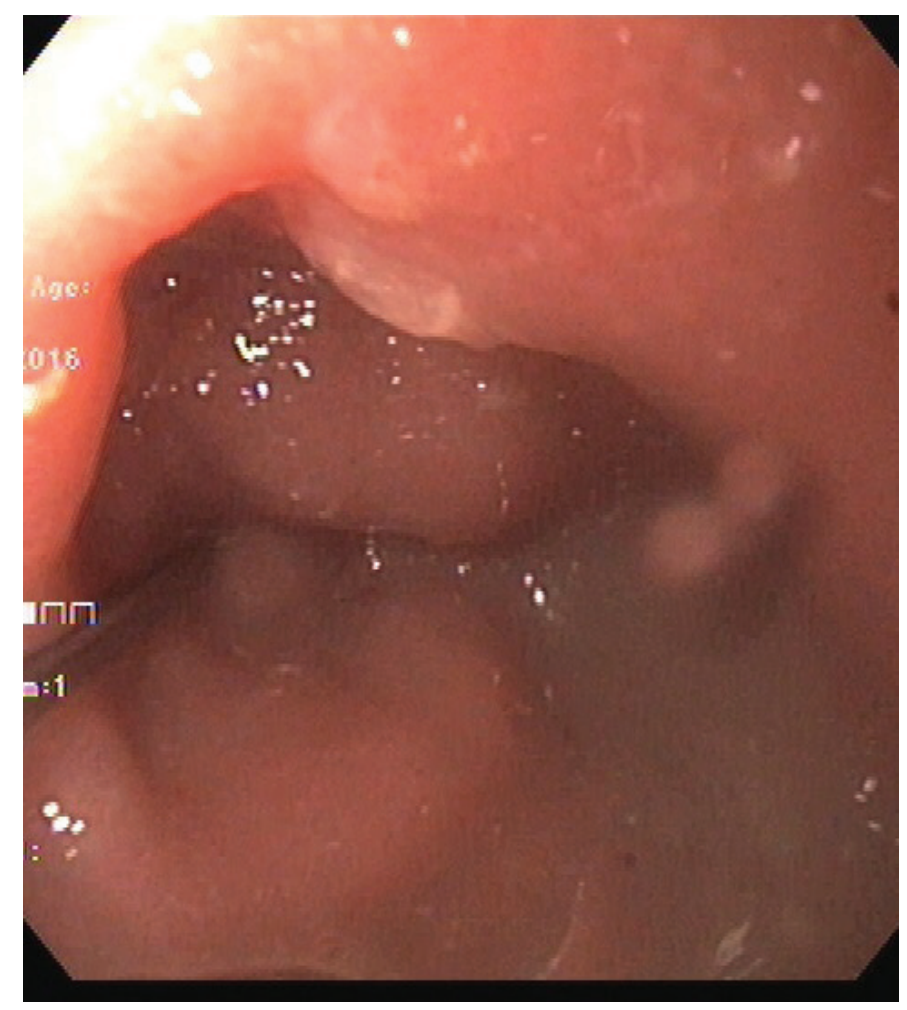

Figure 2: Endoscopy: Migrated FC-SEMS. Steel guide wire in the transluminal tract.

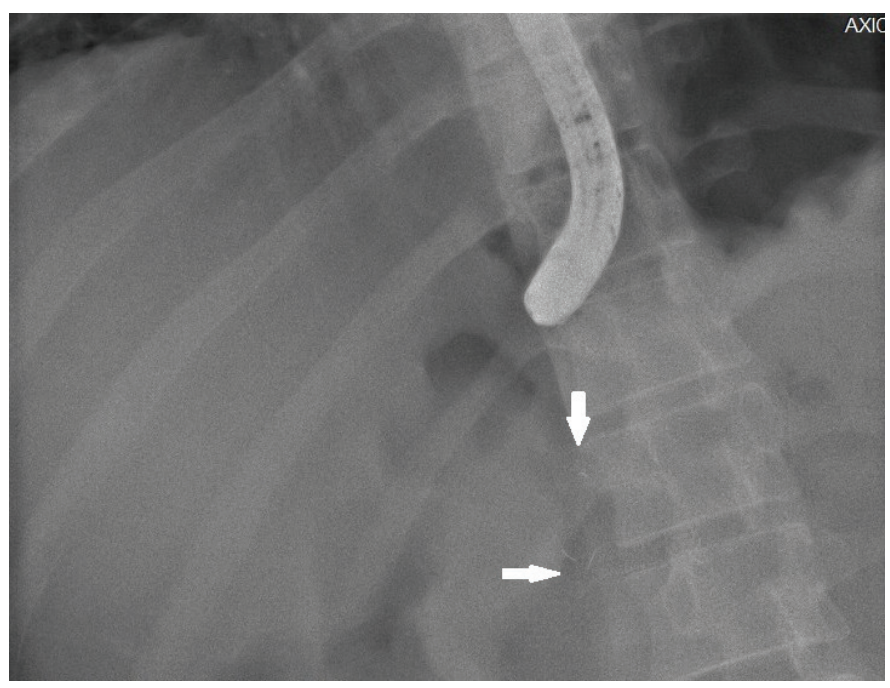

Figure 3: Inward migrated FC-SEMS (arrows). Endoscope tip at the mouth of the transluminal tract.

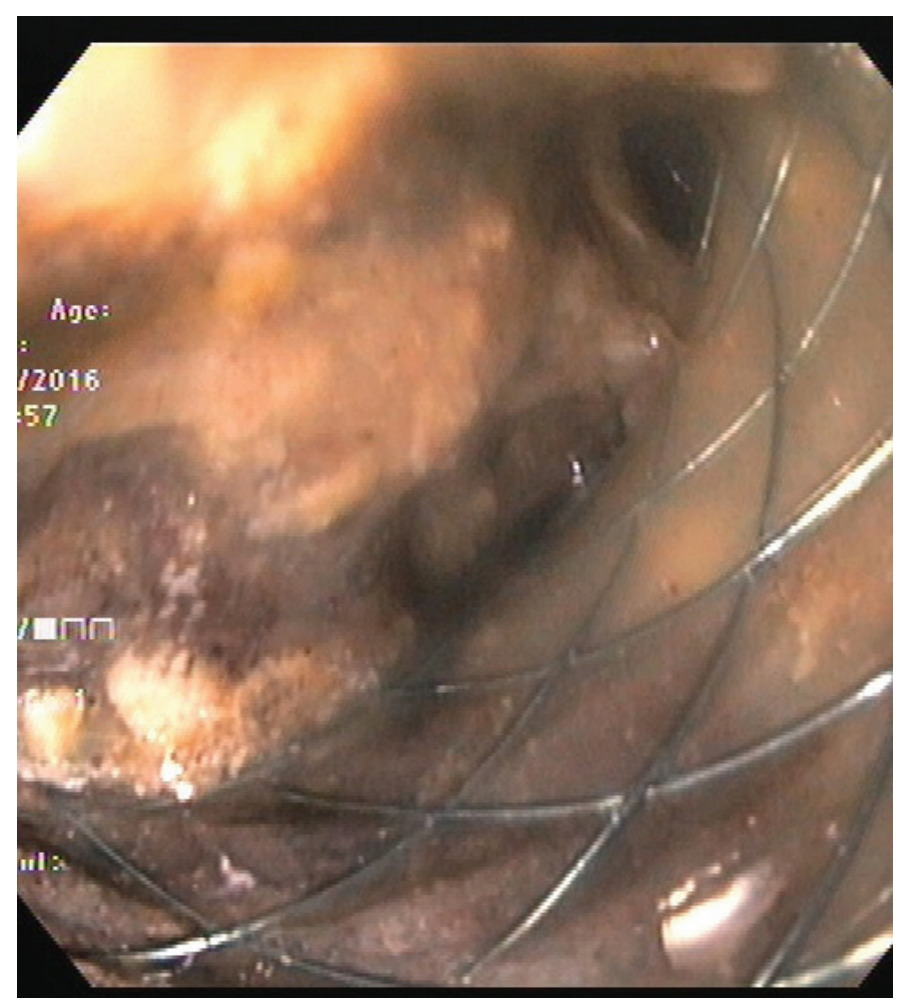

Figure 4: FC-SEMS in WOPN cavity.

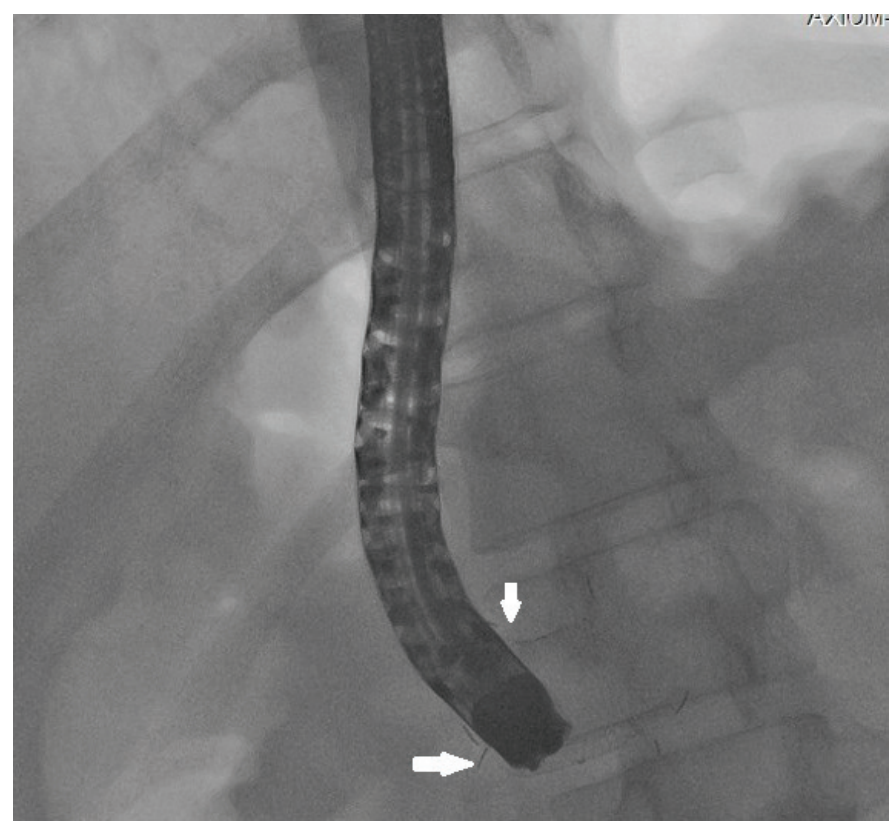

Figure 5: FC-SEMS grasped with rat tooth forceps. Proximal end shown by arrows. 


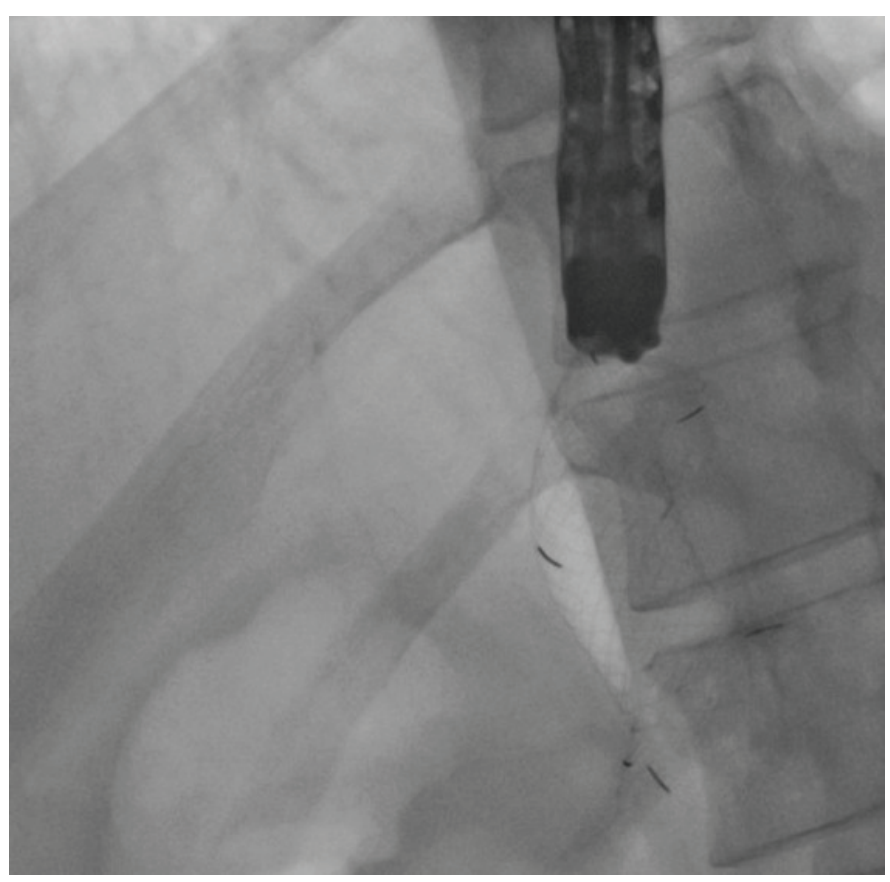

Figure 6: Stent was gradually pulled back.

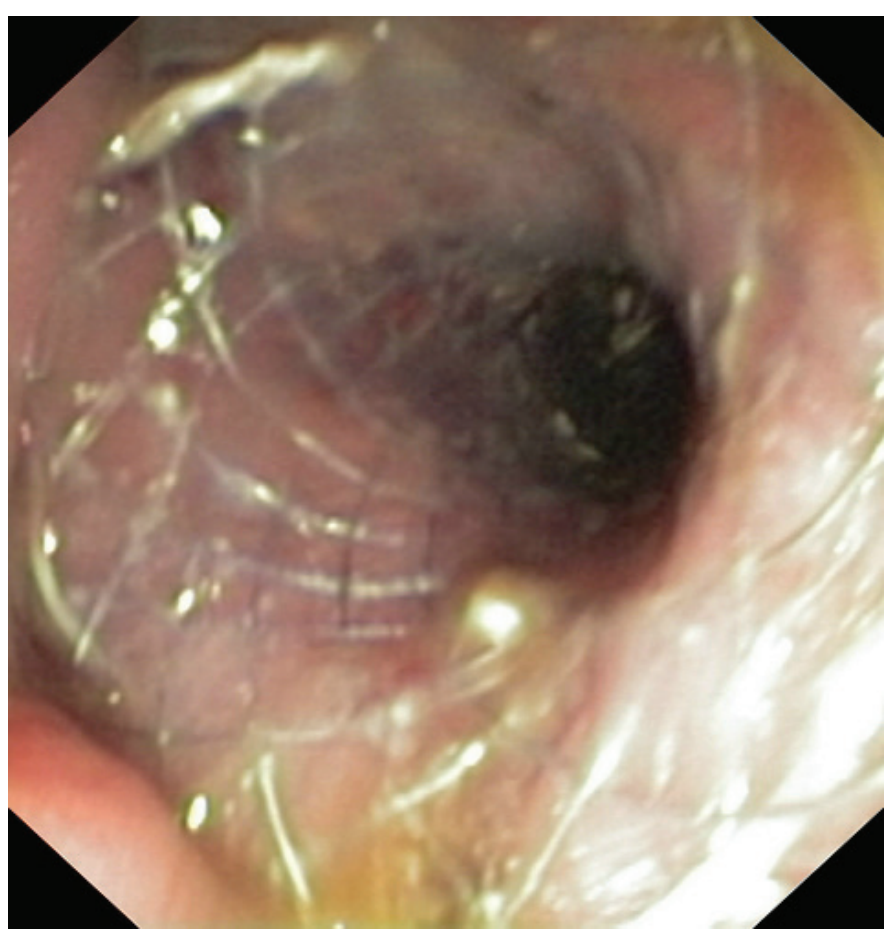

Figure 7: Repositioning of stent across the gastric wall.

\section{Discussion}

Endoscopic transluminal drainage of WOPN involves using more aggressive techniques such as placement of multiple stents, aggressive irrigation using nasocystic catheter, and direct debridement of necrotic tissue. ${ }^{1-5}$ FC-SEMS provide a larger diameter for drainage and also decrease risk of perforation and bleeding because of minimal dilation and sealing of the fistula tract. ${ }^{6}$ The FCSEMS have higher risk of migration but large and acute angled flare ends decrease risk of migration. Migration has been occasionally reported but mostly it is outward and inward migration is very rare, in which case endoscopic recovery is possible. ${ }^{6}$

\section{SURINDER SINGH RANA ${ }^{1}$ RAVI SHARMA ${ }^{1}$ LOVNEET DHALARIA ${ }^{1}$ VISHAL SHARMA ${ }^{1}$ RAJESH GUPTA ${ }^{2}$}

${ }^{1}$ Departments of Gastroenterology \& ${ }^{2}$ Department of Surgery, Post Graduate Institute of Medical Education and Research (PGIMER), Sector 12, Chandigarh - 160012, India.

Correspondence: Surinder Singh Rana Email:drsurinderrana@yahoo.co.in

\section{References}

1. Banks PA, Bollen TL, Dervenis C, Gooszen HG, Johnson $\mathrm{CD}$, Sarr $\mathrm{MG}$, et al. Acute Pancreatitis Classification Working Group. Classification of acute pancreatitis-2012: revision of the Atlanta classification and definitions by international consensus. Gut 2013;62:102-11.

2. Rana SS, Bhasin DK, Rao C,Gupta R, Singh K. Nonfluoroscopic endoscopic ultrasound-guided transmural drainage of symptomatic non-bulging walled-off pancreatic necrosis. Dig Endosc. 2013;25:47-52.

3. Gardner TB, Chahal P, Papachristou GI, Vege SS, Petersen BT, Gostout CJ, et al. A comparison of direct endoscopic necrosectomy with transmural endoscopic drainage for the treatment of walled-off pancreatic necrosis. Gastrointest Endosc. 2009;69:1085-94.

4. Rana SS, Bhasin DK, Sharma RK,Kathiresan J, Gupta R. Do the morphological features of walled-off pancreatic 
necrosis on endoscopic ultrasound determine the outcome of endoscopic transmural drainage? Endosc Ultrasound. 2014;3:118-22.

5. Rana SS, Bhasin DK. Should all fluid collections in delayed phase of acute necrotizing pancreatitis labeled as walledoff pancreatic necrosis? Dig Dis Sci. 2014;59:1338-9.

6. Talreja JP, Shami VM, Ku J, Morris TD, Ellen K, Kahaleh M. Transenteric drainage of pancreatic-fluid collections with fully covered self-expanding metallic stents (with video). Gastrointest Endosc. 2008 ;68:1199-203.

\section{Prevertebral tubercular abscess diagnosed by endoscopic ultrasound-guided fine needle aspiration}

Endoscopic ultrasound (EUS) is an excellent investigational modality for evaluation of various mediastinal pathologies. ${ }^{1,2}$ It is perhaps the most sensitive method for the diagnosis of posterior mediastinal lesions and lymph nodes. ${ }^{3}$ However, complete mediastinal evaluation is not possible with EUS because of its limited ability to visualise the anterior mediastinum because of the presence of the trachea and bronchi anteriorly.

\section{Case Report}

A 38-year old male patient was referred to us for evaluation of ascites of two months' duration. He had a history of significant alcohol consumption and an ultrasound abdomen revealed ascites along with an enlarged liver with an irregular outline and intra abdominal collaterals. There were no varices on gastroscopy and contrast enhanced computed tomography (CECT) of abdomen revealed findings similar to that of ultrasound. Ascitic fluid analysis revealed a high gradient ascites with a high adenosine deaminase value (108 IU/l) and absence of malignant cells. He was started on diuretics and anti tubercular therapy (ATT). However, he continued to require repeated therapeutic paracentesis. Endoscopic ultrasound (EUS) was done to look for peritoneal deposits but none could be identified in the upper peritoneum. A few enlarged celiac lymph nodes were identified and fine needle aspiration (FNA) cytology revealed reactive lymphoid hyperplasia. No significantly enlarged mediastinal lymph nodes were seen but a small pre vertebral abscess was identified in the upper mediastinum (Figure1). An EUS-guided FNA yielded cheesy white material (Figure2) and a cytological examination revealed epithelioid cell granulomas in a background of extensive necrosiswith the presence of acid fast bacilli (Figure3). Magnetic Resonance Imaging of the thoracic spine revealed an altered marrow signal involving the body of the third and fourth thoracic vertebra with a pre-vertebral collection showing heterogeneous enhancement on contrast (Figure4). He was continued on ATT and diuretics and did not require further paracentesis after completion of three weeks of treatment.

\section{Discussion}

EUS FNA is an excellent diagnostic modality for the evaluation of posterior mediastinal lymph nodes as well as soft tissue lesions. ${ }^{1-3}$ Various studies have demonstrated the safety and efficacy of EUS FNA in these diseases. Diseases involving the anterior vertebral space and vertebrae in the mediastinum can be potentially diagnosed by EUS because of the close relation of these structures with the esophagus. However, there are only few reports of diagnosis of various vertebral pathologies by EUS. There have been reports of diagnosis of anterior vertebral osteophytes by EUS but the diagnosis of pre-vertebral tubercular abscess by EUS-guided aspiration has not been previously reported. ${ }^{4,5}$ 